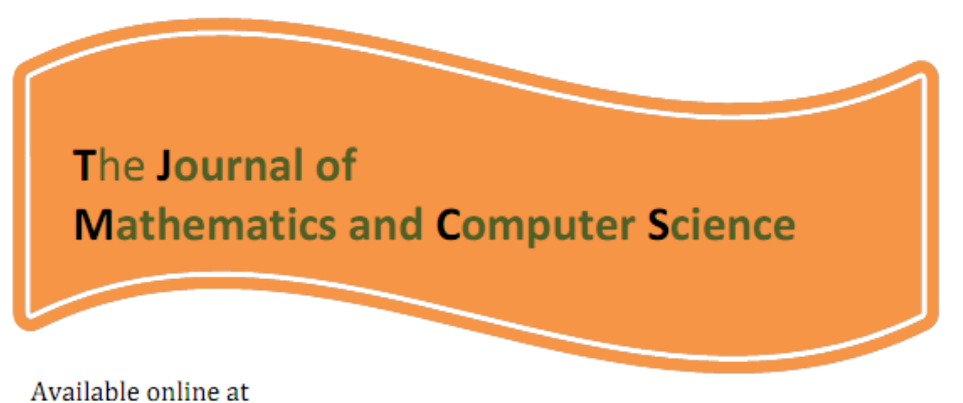

\title{
http://www.TJMCS.com
}

The Journal of Mathematics and Computer Science Vol .3 No.3 (2011) 318 - 328

\section{Solving Linear Programming Problem with Fuzzy Right Hand Sides: A Penalty Method}

\author{
S.H. Nasseri* and Z. Alizadeh \\ Department of Mathematics, University of Mazandaran, Babolsar, Iran
}

Received: August 2011, Revised: November 2011

Online Publication: December 2011

\begin{abstract}
Linear programming problems with trapezoidal fuzzy variables (FVLP) have recently attracted some interest. Some methods have been developed for solving these problems. Fuzzy primal and dual simplex algorithms have been recently proposed to solve these problems. These methods have been developed with the assumption that an initial Basic Feasible Solution (BFS) is at hand. In many cases, finding such a BFS is not straightforward and some works may be needed to get the simplex algorithm started. In this paper, we propose a penalty method to solve FVLP problems in which the BFS is not readily available.
\end{abstract}

\section{Introduction}

Many application problems, modeled as mathematical programming problems, may be formulated with uncertainty. Fuzzy programming approach is useful to treat a problem under

\footnotetext{
*Corresponding author. nasseri@umz.ac.ir (Hadi Nasseri)
} 
uncertainty. The concept of decision making in fuzzy environment was first proposed by Bellman and Zadeh [2]. Several kinds of fuzzy linear programming problems have appeared in the literature and different methods have been proposed to solve such problems. The first formulation of Fuzzy Linear Programming (FLP) is proposed by Zimmermann [12]. Some methods have been developed for solving these problems by introducing and solving certain auxiliary problems. Mahdavi-Amiri et al. [10] have developed a new fuzzy primal and dual simplex algorithm for solving the FVLP problem. As they have been mentioned the fuzzy primal algorithm needs an initial basic feasible solution (see[10]). In some cases, such a solution is not straightforward. Hence in this paper we propose a fuzzy Big-M method for solving these types of problems where directly can find a basic feasible solution if there exist. Our discussion, here is outlined as follows: In next section we briefly give some necessary concepts of fuzzy set theory. In Section 3 we first define a Linear Programming with Fuzzy Variables (FVLP) problem and then formulate a real problem as FVLP problem. Furthermore, the fundamental concepts and theorems are given to complete our discussion in this section. We also introduce a Big-M method for solving FVLP problems and illustrate it with an example in Section 4. Finally, we conclude in Section 5.

\section{Preliminaries}

We review the fundamental notations of fuzzy set theory, initiated by Bellman and Zadeh [2], to be used throughout this note and which is taken from ([5],[6],[8],[9]and[10]). We give here, some basic definitions on fuzzy numbers comparison that will be used for illustrating our approach. In this way, and for methodological reasons, in spite that the approach that is designed will work correctly for any kind of fuzzy numbers, we prefer in the following to focus on the usual case of trapezoidal fuzzy numbers.

Definition 1. Let $X$ be the universal set. $\widetilde{A}$ is called a fuzzy set in $X$ if $\widetilde{A}$ is a set of ordered pairs $\widetilde{A}=\left\{\left(x, \mu_{\widetilde{A}}(x)\right) \mid x \in X\right\}$, where $\mu_{\widetilde{A}}{ }_{\widetilde{A}}^{(x)}$ is the membership function of $x$ in $\widetilde{A}$.

remark 1. The membership function of $\widetilde{A}$ specifies the degree of membership of element $x$ in fuzzy set $\widetilde{A}$ (in fact, $\mu_{\widetilde{A}}$ shows the degree that $x$ belongs to $\widetilde{A}$ ).

Definition 2. The $\alpha$-level set of $\widetilde{A}$ is the set $\widetilde{A}_{\alpha}=\left\{x \in \mathbb{R} \mid \mu_{\widetilde{A}}(x) \geq \alpha\right\}$, where $\alpha \in(0,1]$. The lower and upper bounds of any $\alpha$-level set $\widetilde{A}_{\alpha}$ are represented by finite numbers inf ${ }_{x \in \widetilde{A}_{\alpha}}$ and $\sup _{x \in \widetilde{A}_{\alpha}}$.

Definition 3. The support of a fuzzy set $\widetilde{A}$ is a set of elements in $X$ for which $\mu_{\widetilde{A}}(x)$ is positive, that is, supp $\widetilde{A}=\left\{x \in X \mid \mu_{\widetilde{A}}(x)>0\right\}$. 
Definition 4. A fuzzy set $\widetilde{A}$ is convex if

$$
\mu_{\widetilde{A}}(\lambda x+(1-\lambda) y) \geq \min \left\{\mu_{\widetilde{A}}(x), \mu_{\widetilde{A}}(y)\right\}, \quad \forall x, y \in X \text { and } \lambda \in[0,1] .
$$

Definition 5. A convex fuzzy set $\widetilde{A}$ on $\mathbb{R}$ is a fuzzy number if the following conditions hold: (a)Its membership function is piecewise continuous.

(b) There exist three intervals $[a, b],[b, c]$ and $[c, d]$ such that $\mu_{\widetilde{A}}$ is increasing on $[a, b]$, equal to 1 on $[b, c]$, decreasing on $[c, d]$ and aqual to 0 elsewhere.

remark 2. In the above definition, we say interval $[b, c]$ is the modal set of fuzzy number $\widetilde{A}$.

Definition 6. Let $\widetilde{A}=\left(a^{L}, a^{U}, \alpha, \beta\right)$ denote the trapezoidal fuzzy number, where $\left[a^{L}-\alpha, a^{U}+\beta\right]$ is the support of $\widetilde{A}$ and $\left[a^{L}, a^{U}\right]$ its modal set.

remark 3. We denote the set of all trapezoidal fuzzy numbers by $F(R)$. If $a=a^{L}=a^{U}$ then we obtain a triangular fuzzy number, and we show it with $\widetilde{A}=(a, \alpha, \beta)$.

\subsection{Arithmetic on fuzzy numbers}

Let $\widetilde{a}=\left(a^{L}, a^{U}, \alpha, \beta\right)$ and $\widetilde{b}=\left(b^{L}, b^{U}, \gamma, \theta\right)$ be two trapezoidal fuzzy numbers. Define

$x>0, x \in \mathbb{R} ; \quad x . \widetilde{a}=\left(x a^{L}, x a^{U}, x \alpha, x \beta\right)$,

$x<0, x \in \mathbb{R} ; \quad x . \widetilde{a}=\left(x a^{U}, x a^{L},-x \beta,-x \alpha\right)$,

$\widetilde{a}+\widetilde{b}=\left(a^{L}+b^{L}, a^{U}+b^{U}, \alpha+\gamma, \beta+\theta\right)$,

$\widetilde{a}-\widetilde{b}=\left(a^{L}-b^{U}, a^{U}-b^{L}, \alpha+\theta, \beta+\gamma\right)$.

\subsection{Ranking function}

An effective approach for ordering the elements of $F(R)$ is also to define a ranking function $R: F(R) \rightarrow \mathbb{R}$ which maps each fuzzy number into the real line, where a natural order exists. We define orders on $F(R)$ by:

$$
\begin{aligned}
& \widetilde{a} \succeq \widetilde{b} \text { if and only if } R(\widetilde{a}) \geq R(\widetilde{b}) \\
& \widetilde{a} \succ \widetilde{b} \text { if and only if } R(\widetilde{a}) \geq R(\widetilde{b}) \\
& \widetilde{a} \cong \widetilde{b} \text { if and only if } R(\widetilde{a})=R(\widetilde{b})
\end{aligned}
$$

where $\widetilde{a}$ and $\widetilde{b}$ are in $F(R)$. Also we write $\widetilde{a} \preceq \widetilde{b}$ if and only if $\widetilde{b} \succeq \widetilde{a}$.

We restrict our attention to linear ranking functions, that is, a ranking function $R$ such that 


$$
R(k \widetilde{a}+\widetilde{b})=k R(\widetilde{a})+R(\widetilde{b})
$$

for any $\widetilde{a}$ and $\widetilde{b}$ belonging to $F(R)$ and any $k \in R$.

We consider the linear ranking functions on $F(R)$ as:

$$
R(\widetilde{a})=c_{L} a^{L}+c_{U} a^{U}+c_{\alpha} \alpha+c_{\beta} \beta
$$

where $\widetilde{a}=\left(a^{L}, a^{U}, \alpha, \beta\right)$, and $c_{L}, c_{U}, c_{\alpha}, c_{\beta}$ are constants, at least one of which is nonzero. $A$ special version of the above linear ranking function was first proposed by Yager[11] as follows:

$$
R(\widetilde{a})=\frac{1}{2} \int_{0}^{1}\left(\inf \widetilde{a}_{\lambda}+\sup \widetilde{a}_{\lambda}\right) d \lambda
$$

which reduces to

$$
R(\widetilde{a})=\frac{a^{L}+a^{U}}{2}+\frac{1}{4}(\beta-\alpha) .
$$

Then, for trapezoidal fuzzy numbers $\widetilde{a}=\left(a^{L}, a^{U}, \alpha, \beta\right)$ and $\widetilde{b}=\left(b^{L}, b^{U}, \gamma, \theta\right)$, we have

$$
\widetilde{a} \succeq \widetilde{b} \quad \text { if and only if } \quad a^{L}+a^{U}+\frac{1}{2}(\beta-\alpha) \geq b^{L}+b^{U}+\frac{1}{2}(\theta-\gamma) .
$$

\section{$3 \quad$ Fuzzy linear programme}

\subsection{Definition of model}

Here we first define a general form of the problem which will be discussed in this paper. Consider a Linear Programming with Fuzzy Variables (FVLP) as follows:

$$
\begin{aligned}
\min \quad \widetilde{z} & \approx c \widetilde{x} \\
\text { s.t. } & A \widetilde{x} \succeq \widetilde{b} \\
& \widetilde{x} \succeq \widetilde{0}
\end{aligned}
$$

where $c \in \mathbb{R}^{n}, \widetilde{x} \in(F(\mathbb{R}))^{n}, A \in \mathbb{R}^{m \times n}, \widetilde{b} \in(F(\mathbb{R}))^{m}$ and $\widetilde{b} \succeq \widetilde{0}$.

Note that an FVLP problem is a linear programming problem in fuzzy environment in which the decision making variables and right-hand sides are fuzzy numbers. 


\subsection{Formulation of a problem}

An alloy producer, produces 2 types of alloys $P_{1}$ and $P_{2}$. These alloys consists of Zinc and $\operatorname{Tin}\left(F_{1}, F_{2}\right)$. The tableau below shows the amount of $F_{1}$ and $F_{2}$ used in per unit of alloys. The average cost of per kilogram of $P_{1}$ and $P_{2}$ are 10 and 6 cents, respectively. The minimum daily supplyes metals $F_{1}$ and $F_{2}$ are approximately 49 and 45 units, respectively. The producer wants to know in order to minimize the cost of Zinc and Tin how many kilograms of alloys $P_{1}$ and $P_{2}$ he must produce daily?

\begin{tabular}{|c|cc|}
\hline & \multicolumn{2}{|c|}{ Products } \\
\hline Metal & $P_{1}$ & $P_{2}$ \\
\hline$F_{1}$ & 1 & 6 \\
\hline$F_{2}$ & 4 & 2 \\
\hline
\end{tabular}

Now if we assume that the fuzzy variables $\widetilde{x}_{1}$ and $\widetilde{x}_{2}$ be the daily supplyes metals $F_{1}$ and $F_{2}$ respectively, then this problem can be formulated as follows:

$$
\begin{gathered}
\min \widetilde{z} \approx 10 \widetilde{x}_{1}+6 \widetilde{x}_{2} \\
\text { s.t. } \quad \widetilde{x}_{1}+6 \widetilde{x}_{2} \succeq(46,52,2,2) \\
4 \widetilde{x}_{1}+2 \widetilde{x}_{2} \succeq(42,48,4,4) \\
\widetilde{x}_{1}, \widetilde{x}_{2} \succeq \widetilde{0}
\end{gathered}
$$

\subsection{Fuzzy basic feasible solution}

First of all, we briefly describe Fuzzy Basic Feasible Solution (FBFS) for the FVLP problem as established by Mahdavi-Amiri and Nasseri [8]. Consider the FVLP problem and $A=\left[a_{i j}\right]_{m \times n}$. Assume $\operatorname{rank}(A)=m$. Partition $A$ as $\left[\begin{array}{ll}B & N\end{array}\right]$ where $B, m \times m$, is nonsingular. It is obvious that $\operatorname{rank}(B)=m$. Let $y_{j}$ be the solution to $B y=a_{j}$. It is apparent that the basic solution $\widetilde{x}_{B} \approx\left(\widetilde{x}_{B_{1}}, \ldots, \widetilde{x}_{B_{m}}\right)^{T} \approx B^{-1} \widetilde{b}, \widetilde{x}_{N} \approx \widetilde{0}$ is a solution of $A \widetilde{x}=\widetilde{b}$. In fact, $\widetilde{x}=\left(\begin{array}{lll}\widetilde{x}_{B}^{T} & \widetilde{x}_{N}^{T}\end{array}\right)^{T}$. If $\widetilde{x}_{B} \succeq \widetilde{0}$, then the basic solution is feasible and the corresponding fuzzy objective value is: $\widetilde{z} \approx c_{B} \widetilde{x}_{B}$, where $c_{B}=\left(c_{B_{1}}, \ldots, c_{B_{m}}\right)$. Now, corresponding to every nonbasic variable $\widetilde{x}_{j}, 1 \leq j \leq n, j \neq B_{i}, i=1, \ldots, m$, define $z_{j}=c_{B} y_{j}=c_{B} B^{-1} a_{j}$. The following result concerns the non-degenerated problems, where every fuzzy basic variable corresponding to every basis $B$ is positive. Observe that for any basic index $j=B_{i}, 1 \leq i \leq m$, we have $B^{-1} a_{j}=e_{i}$ where $e_{i}=(0, \ldots, 0,1,0, \ldots, 0)^{T}$ is the ith unit vector, since $B e_{i}=\left[a_{B_{1}}, \ldots, a_{B_{i}}, \ldots, a_{B_{m}}\right] e_{i}=a_{B_{i}}=a_{j}$, and so we have: 


$$
z_{j}-c_{j}=c_{B} B^{-1} a_{j}-c_{j}=c_{B} e_{i}-c_{j}=c_{B_{i}}-c_{j}=c_{j}-c_{j}=0 .
$$

The following theorem characterizes optimal solutions.

Theorem 1. (optimality conditions) Assume the FVLP problem is non-degenerate. If a basic solution $\widetilde{x}_{B}=B^{-1} \widetilde{b}, \quad \widetilde{x}_{N} \approx \widetilde{0}$ is feasible to (1) and $z_{j} \geq c_{j}$ for all $j, 1 \leq j \leq n$ then the fuzzy basic solution is a fuzzy optimal solution to (1).

Proof: See Mahdavi-Amiri and Nasseri [8].

Theorem 2. Let $\widetilde{x}_{B}=B^{-1} \widetilde{b}$ is a fuzzy basic feasible solution of the problem max $\widetilde{z} \approx c \widetilde{x}$ such that $A \widetilde{x} \approx \widetilde{b}$ and $\widetilde{x} \succeq \widetilde{0}$. If for any column $a_{j}$ in $A$ which is not in $B$, the condition $\left(z_{j}-c_{j}\right)<0$ hold and $y_{i j}>0$ for some $i, j \in\{1,2, \ldots, m\}$ then it is possible to obtain a new fuzzy basic feasible solution by replacing one of the columns in $B$ by $a_{j}$.

Proof: See[8].

Theorem 3. If in an FVLP problem, there is a column $a_{j}$ in $A$ (not in basis) for which $z_{j}-c_{j}<0$ and $y_{i j} \leq 0, i=1, \ldots, m$, then the FVLP problem is unbounded.

Proof: It is straight forward.

We write the above FVLP problem in the following tableau format:

\begin{tabular}{|c|cc|c|}
\hline Basis & $\tilde{x}_{B}$ & $\tilde{x}_{N}$ & R.H.S. \\
\hline$\widetilde{z}$ & 0 & $z_{N}-c_{N}=c_{B} B^{-1} N-c_{N}$ & $\widetilde{y}_{00}=c_{B} B^{-1} \vec{b}$ \\
\hline$\widetilde{x}_{B}$ & $I$ & $Y=B^{-1} N$ & $\widetilde{y}_{0}=B^{-1} \widetilde{b}$ \\
\hline
\end{tabular}

Now we are going to give the concept of pivoting and change of basis.

If $\widetilde{x}_{k}$ enters the basis and $\widetilde{x}_{B_{r}}$ leaves the basis, then pivoting on $y_{r k}$ in the simplex tableau is stated as follows:

1. Divide row $r$ by $y_{r k}$

2. For $i=0,1, \ldots, m$ and $i \neq r$, update the $i$ th row by adding to it $-y_{i k}$ times the new $r$ th row.

\section{Big-M Method}

The simplex algorithm was conceived by Dantzig for solving Linear Programming (LP)problems. This method starts with a Basic Feasible Solution (BFS) and moves to an improved BFS, until the optimal point is reached or else unboundedness of the objective function is verified. 
In order to initialize this algorithm a BFS must be available. In many cases, finding such a BFS is not straightforward and some work may be needed to get the simplex algorithm started.To this end, there are two techniques in linear programming literature: Two-phase method and Big-M method [1]. But there may be some LP models for which there are not any BFSs, i.e., the model is infeasible. Both Two-phase method and Big-M method distinguish the infeasibility. Here we focus on Big-M method.

Consider a generic LP model. After manipulating the constraints and introducing the required slack variables, the constraints are put in the format $A X=b, X \geq 0$ where $A$ is a $m \times n$ matrix and $b \geq 0$ an $m \times 1$ vector. Considering $C$ as cost vector, the following $L P$ model is dealt with:

$$
\begin{gathered}
\min C X \\
\text { s.t. } \quad A X=b \\
\\
X \geq 0
\end{gathered}
$$

Furthermore, suppose that we do not have a starting BFS for simplex metod, i.e, A has no identity submatrix. In this case we shall resort to the artificial variables to get a starting BFS, and then use the simplex method itself and get rid of these artificial variables. The use of artificial variables to obtain a starting BFS was first provided by Dantzig [3, 4].

To illustrate, suppose that we change the restrictions by adding an artificial vector $R$ leading to the system $A X+R=b,(X, R) \geq 0$. This forces an identity submatrix corresponding to the artificial vector and gives an immediate BFS of the new system, namely $(X=0, R=b)$. Even though we now have a starting BFS and the simplex method can be applied, we have in effect changed the problem. In order to get back to our original problem, we must force these artificial variables to zero, because $A X=b \Leftrightarrow A X+R=b, R=0$. In Big-M method we assign a large penalty coefficient to these variables in the original objective function in such a way as to make their presence in the basis at a positive level very unattractive from the objective function point of view. More specifically, $(P)$ is changed to:

$$
\begin{array}{ll}
\min & C X+M R \quad P(M) \\
\text { s.t. } & A X+R=b \\
& (X, R) \geq 0
\end{array}
$$


where $M$ is a very large positve number. The term $M R$ can be interpreted as a penalty to be paid by any solution with $R \neq 0$. Therefore the simplex method itself will try to get the artificial variables out of the basis, and then continue to find an optimal solution of the original problem. Hereafter '*' indicates the optimality, $z_{j}-c_{j}$ is the reduced cost of the $j$ th variable, and $y_{j}=B^{-1} a_{j}$, where $B$ is the basis of the simplex method associated with the related iteration and $a_{j}$ is the $j$ th column of the technological coefficients matrix (for $P(M)$ it is $\left.\left[\begin{array}{ll}A & I\end{array}\right]\right)$. Four possible cases may arise while solving $P(M)$ :

$\left(A_{1}\right):\left(X^{*}, R^{*}\right)$ is an optimal solution of $P(M)$, in which $R^{*}=0$

$\left(A_{2}\right):\left(X^{*}, R^{*}\right)$ is an optimal solution of $P(M)$, in which $R^{*} \neq 0$

$\left(B_{1}\right): z_{k}-c_{k}=\max \left(z_{j}-c_{j}\right)>0, y_{k} \leq 0$, and all artificials are equal to zero.

$\left(B_{2}\right): z_{k}-c_{k}=\max \left(z_{j}-c_{j}\right)>0, y_{k} \leq 0$, and not all artificials are equal to zero.

remark 4. A Two-phase method for solving FVLP problem was proposed later [7]. Here we are going to solve an FVLP problem using Big-M method.

Consider the following problem:

$$
\begin{gathered}
\min \widetilde{Z}=10 \widetilde{x}_{1}+6 \widetilde{x}_{2} \\
\text { s.t. } \widetilde{x}_{1}+6 \widetilde{x} \geq(46,52,2,2) \\
4 \widetilde{x}_{1}+2 \widetilde{x}_{2} \geq(42,48,4,4) \\
\widetilde{x}_{1}, \widetilde{x}_{2} \succeq \widetilde{0}
\end{gathered}
$$

First of all we add the slack variables:

$$
\begin{gathered}
\min \widetilde{Z}=10 \widetilde{x}_{1}+6 \widetilde{x}_{2} \\
\text { s.t. } \\
\widetilde{x}_{1}+6 \widetilde{x}-\widetilde{x}_{3}=(46,52,2,2) \\
4 \widetilde{x}_{1}+2 \widetilde{x}_{2}-\widetilde{x}_{4}=(42,48,4,4) \\
\widetilde{x}_{1}, \widetilde{x}_{2}, \widetilde{x}_{3}, \widetilde{x}_{4} \succeq \widetilde{0}
\end{gathered}
$$

As its seen no initial basic variable is available and hence we may use the Big-M method to solve the problem.

Therefore, the problem changes to the following form:

$$
\begin{aligned}
& \min \widetilde{Z}=10 \widetilde{x}_{1}+6 \widetilde{x}_{2}+M \widetilde{R}_{1}+M \widetilde{R}_{2} \\
& \text { s.t. } \widetilde{x}_{1}+6 \widetilde{x}-\widetilde{x}_{3}+\widetilde{R}_{1}=(46,52,2,2)
\end{aligned}
$$




$$
\begin{gathered}
4 \widetilde{x}_{1}+2 \widetilde{x}_{2}-\widetilde{x}_{4}+\widetilde{R}_{2}=(42,48,4,4) \\
\widetilde{x}_{1}, \widetilde{x}_{2}, \widetilde{x}_{3}, \widetilde{x}_{4}, \widetilde{R}_{1}, \widetilde{R}_{2} \succeq \widetilde{0}
\end{gathered}
$$

The problem is written in the tableau format as:

\begin{tabular}{|c|cccccc|c|}
\hline Basis & $\tilde{x}_{1}$ & $\tilde{x}_{2}$ & $\tilde{x}_{3}$ & $\tilde{x}_{4}$ & $\tilde{R}_{1}$ & $\tilde{R}_{2}$ & R.H.S. \\
\hline$\widetilde{z}$ & -10 & -6 & 0 & 0 & $-M$ & $-M$ & 0 \\
\hline$\widetilde{R}_{1}$ & 1 & 6 & -1 & 0 & 1 & 0 & $(46,52,2,2)$ \\
\hline$\widetilde{R}_{2}$ & 4 & 2 & 0 & -1 & 0 & 1 & $(42,48,4,4)$ \\
\hline
\end{tabular}

Because $\widetilde{R}_{1}$ and $\widetilde{R}_{2}$ are basic variables, so the cost row must be equal to zero. Hence the next tableau will be as following:

\begin{tabular}{|c|cccccc|c|}
\hline Basis & $\tilde{x}_{1}$ & $\tilde{x}_{2}$ & $\tilde{x}_{3}$ & $\tilde{x}_{4}$ & $\tilde{R}_{1}$ & $\tilde{R}_{2}$ & R.H.S. \\
\hline$\widetilde{z}$ & $-10+5 M$ & $-6+8 M$ & $-M$ & $-M$ & 0 & 0 & $(88 M, 100 M, 6 M, 6 M)$ \\
\hline$\widetilde{R}_{1}$ & 1 & 6 & -1 & 0 & 1 & 0 & $(46,52,2,2)$ \\
\hline$\widetilde{R}_{2}$ & 4 & 2 & 0 & -1 & 0 & 1 & $(42,48,4,4)$ \\
\hline
\end{tabular}

It is obvious that $\widetilde{x}_{2}$ is an entering fuzzy variable and $\widetilde{R}_{1}$ is a leaving fuzzy variable. Then after pivoting the next tableau is given as:

\begin{tabular}{|c|cccccc|c|}
\hline Basis & $\tilde{x}_{1}$ & $\tilde{x}_{2}$ & $\tilde{x}_{3}$ & $\tilde{x}_{4}$ & $\tilde{R}_{1}$ & $\tilde{R}_{2}$ & R.H.S. \\
\hline$\widetilde{z}$ & $-9+\frac{11}{3} M$ & 0 & $\frac{M}{3}-1$ & $-M$ & $1-\frac{4}{3} M$ & 0 & $\left(48+\frac{74 M}{3}, 52+\frac{98 M}{3}, 2+\frac{14 M}{3}, 2+\frac{14 M}{3}\right)$ \\
\hline$\widetilde{x}_{2}$ & $\frac{1}{6}$ & 1 & $\frac{-1}{6}$ & 0 & $\frac{1}{6}$ & 0 & $\left(\frac{46}{6}, \frac{52}{6}, \frac{2}{6}, \frac{2}{6}\right)$ \\
\hline$\widetilde{R}_{2}$ & $\frac{11}{3}$ & 0 & $\frac{1}{3}$ & -1 & $\frac{-1}{3}$ & 1 & $\left(\frac{74}{3}, \frac{98}{3}, \frac{14}{3}, \frac{14}{3}\right)$ \\
\hline
\end{tabular}

So $\widetilde{x}_{1}$ is an entering fuzzy variable and $\widetilde{R}_{2}$ is a leaving fuzzy variable. The last tableau is given in the below. Now we can see all artificial variables leaves the basis. So we can remove their columns

\begin{tabular}{|c|cccccc|c|}
\hline Basis & $\tilde{x}_{1}$ & $\tilde{x}_{2}$ & $\tilde{x}_{3}$ & $\tilde{x}_{4}$ & $\tilde{R}_{1}$ & $\tilde{R}_{2}$ & R.H.S. \\
\hline$\widetilde{z}$ & 0 & 0 & $\frac{-2}{11}$ & $\frac{-27}{11}$ & $\frac{2}{11}-M$ & $\frac{27}{11}-M$ & $\left(\frac{1148}{11}, \frac{1478}{11}, \frac{176}{11}, \frac{176}{11}\right)$ \\
\hline$\widetilde{x}_{2}$ & 0 & 1 & $\frac{-2}{11}$ & $\frac{1}{22}$ & $\frac{2}{11}$ & $\frac{-1}{22}$ & $\left(\frac{68}{11}, \frac{83}{11}, \frac{6}{11}, \frac{6}{11}\right)$ \\
\hline$\widetilde{x}_{1}$ & 1 & 0 & $\frac{1}{11}$ & $\frac{-3}{11}$ & $\frac{-1}{11}$ & $\frac{3}{11}$ & $\left(\frac{74}{11}, \frac{98}{11}, \frac{14}{11}, \frac{14}{11}\right)$ \\
\hline
\end{tabular}

Therefore, the fuzzy optimal solution of the FVLP problem which is obtained by the Big-M method is $\widetilde{x}_{1}=\left(\frac{74}{11}, \frac{98}{11}, \frac{14}{11}, \frac{14}{11}\right), \widetilde{x}_{2}=\left(\frac{68}{11}, \frac{83}{11}, \frac{6}{11}, \frac{6}{11}\right)$ and the fuzzy optimal value of its objective function is $\widetilde{z}=\left(\frac{1148}{11}, \frac{1478}{11}, \frac{176}{11}, \frac{176}{11}\right)$. 


\section{Conclusions}

The Big-M method is a known auxiliary technique to start the simplex algorithm. In particular, we have illustrated the mentiond method by formulating and solving a linear programming problem with fuzzy variables.

\section{Acknowledgment}

The authors would like to thank from the anonymous referees for their valuable comments to improve the earlier version of this paper.

\section{References}

[1] M.S. Bazarra, J.J. Jarvis and H.D. Sherali, Linear Programming and Network Flows, John Wiley and Sons, 1990.

[2] R.E. Bellman, L.A. Zadeh, Decision making in a fuzzy environment, Manag. Sci. 17(1970) 141-164.

[3] G.B. Dantzig, Maximization of a linear function of variables subject to linear inequalities, T.C. Koopmans(Ed.), Activity Analysis of Production and Allocation, John Wiley and Sons, New York, 1951, pp. 339-34\%.

[4] G.B. Dantzig, Programming in a linear structure, Comptroller, United Air Force, Washington, D.C., February, 1948.

[5] D. Dubois, H. Prade, Fuzzy Sets and Systems, Theory and Applications, Academic Press, New York, 1980.

[6] A. Ebrahimnejad, S.H. Nasseri and S.M. Mansourzadeh, Bounded Primal Simplex Algorithm for Bounded Linear Programming with Fuzzy Cost Coefficients, International Journal of Operations Research and Information Systems, 2(1) January-March 2011, 96-120.

[7] B. Khabiri, S.H. Nasseri and Z. Alizadeh, Starting fuzzy solution for the fuzzy primal simplex algorithm using a fuzzy two-phase method, The 5th International Conference on fuzzy Information and Engineering (ICFIE), Oct.14-16, 2011, Chengdu, China. 
[8] N. Mahdavi-Amiri and S.H. Nasseri, Duality results and a dual simplex method for linear programming problems with trapezoidal fuzzy variables, Fuzzy Sets and Systems 158(2007)1961-1978.

[9] N. Mahdavi-Amiri and S.H. Nasseri, Duality in fuzzy number linear programming by use of a certain linear ranking function, Applied Mathematics and Computation 180 (2006)206-216.

[10] N. Mahdavi-Amiri, S.H. Nasseri and A. Yazdani, Fuzzy primal simplex algorithms for solving fuzzy linear programming problems, Iranian Journal of Operational Research 1(2),(2009)68-84.

[11] R.R.Yager, A procedure for ordering fuzzy subsets of the unit interval, Information Sciences 24, (1981)143-161.

[12] H.J. Zimmermann, Fuzzy programming and linear programming with several objective functions, Fuzzy Sets and Systems 1, (1978) 45-55. 